\title{
Prevention of cardiovascular diseases: a spearhead for control of noncommunicable diseases
}

\author{
Pekka Puska
}

After the Second World War, Western countries were faced with a growing burden of cardiovascular disease (CVD) and cancer, seen then as "diseases of affluence". Classical studies soon identified a few strong and obviously causal risk factors for CVD, especially high serum cholesterol, high blood pressure and smoking (1). Smoking was shown as strong cause of many cancers. It was realized that unlike previous public health problems with communicable diseases, the risk factors are strongly related to certain behaviours.

This research opened the possibilities for prevention, and several preventive trials were started in the United States of America (USA) and Europe. Since the behavioural risk factors strongly reflect lifestyles and their cultural and environmental determinants, it became obvious that any major success from the public health point of view would call for interventions in whole communities.

Such programmes were, indeed, started in the 1970s and 1980s, first in the USA and Europe (2). Because Finland was faced with an exceptionally high CVD burden, the North Karelia Project was started there, and its longterm experiences and comprehensive evaluation are well known (3). Later, community-based programmes were also launched in several developing countries, for example the Isfahan Healthy Heart Programme in the Islamic Republic of Iran (4).

An important development was the recognition that the behavioural risk factors for CVD are also risk factors for many other noncommunicable diseases (NCDs). Therefore, many community-based programmes began to adopt an "integrated approach to NCD prevention". WHO coordinates many of these programmes, such as the Countrywide Integrated Noncommunicable Diseases Intervention (CINDI) programme in Europe and CARMEN (Actions for the Multifactorial Reduction of Noncommunicable Diseases) in the Americas (5). The experiences of programmes have varied but many have contributed to national preventive actions, as seen in Finland.

As we entered the 21 st century, the possibilities and potential for CVD prevention had become clear. At the same time, there has been a rapid change in the global public health situation, with NCDs escalating and currently responsible for about two-thirds of all deaths in the world, half of them due to CVD (6). All this formed the background for the pioneering WHO Global Strategy for the Prevention and Control of NCDs in 2000 (7) and subsequent WHO documents and strategies, which highlighted the integrated prevention of CVD, cancers, chronic lung disease and diabetes by intervening on the four key behaviours: unhealthy diet, physical inactivity and tobacco use and harmful use of alcohol.

In the past few years there has been considerable progress in prevention and control of CVDs. Clinical research on diagnostic and therapeutic possibilities has advanced greatly and benefitted patients with these diseases. Although this is welcome, the increasing costs of clinical medicine place a substantial burden on the health services. And the treatment of chronic diseases is often late, especially with the high proportion of sudden cardiovascular deaths. From the public health point of view, good primary health care with evidencebased but inexpensive interventions, as advocated by WHO, have the greatest impact.

Progress in prevention has continued in two particular areas: high-risk and population-based prevention. The high-risk approach aims at detecting persons with high CVD risk and effectively reducing their risk. In this approach, the key is the detection and accurate assessment of the risk. Early emphasis on individual risk factors has been replaced by assessment of total risk, and many scores have been developed for effective risk assessment, e.g. The Framingham or the European risk scores (8).

Although intervention for a high-risk patient may save his or her life, the direct public health impact of this approach is, at best, limited. Thus, population-based prevention has received increasing attention. Both theory and practice show how the population approach has the greatest potential (9). For instance, in Finland, the approximately $80 \%$ reduction in annual CVD mortality in the population under 75 years of age over 30 years and the prevention of some quarter of a million deaths from CVDs during this time in a nation of five million people could never have been achieved through clinical measures (3). 
There are many other advantages of the population approach in controlling CVDs. Since the work is based to a great extent on policies and health promotion, it is cheap compared with the high costs of treatment and high-risk interventions. Prevention through changing lifestyles is thus the cost-effective and sustainable way of reducing the CVD burden. Such national interventions can have an impact on several major NCDs and on public health as a whole, and as such can contribute to favourable social and economic development.

With this background, global political attention on NCD prevention has greatly increased in recent years. This culminated in the High-Level Meeting of the General Assembly on the Prevention and Control of Noncommunicable Diseases in 2011, which resulted in a political declaration (10). Following the UN declaration and based on decisions with the Member States, WHO has prepared a Global NCD Action Plan 2013-2020, with global targets, indicators and intersectoral coordination (6).

The global target is a $25 \%$ reduction in avoidable NCD mortality by 2025. To achieve this, there are targets to change diets (especially concerning salt and quality of fat), to increase physical activity, and to reduce tobacco use and harmful alcohol use. The lifestyle changes should be reflected in favourable changes in the main biological risk factors: blood pressure, blood cholesterol and body weight - all central factors behind CVDs. The action plan also outlines important elements of surveillance, as well as aspects of improved treatment, advocacy, governance and research.

Since CVD accounts for approximately half of all preventable NCD deaths, it is clear that success or failure in achieving the $\mathrm{NCD}$ prevention target depends on success in CVD prevention. Many analyses show that the greatest potential for CVD prevention is in achieving dietary changes in fat, salt and sugar consumption $(11,12)$. It has also been shown recently that reaching the global risk factor targets by 2025 will not quite result in achieving the overall target, but in achieving the CVD target (13).

This is naturally good news, but we must recognize that reaching the targets in many parts of the world will be hard. And to achieve the overall NCD target and further CVD reductions, a more ambitious tobacco reduction target might be warranted and feasible, in view of the many recent achievements in tobacco reduction and with full implementation of the WHO Framework Convention on Tobacco Control.

\section{References}

1. Relationship of blood pressure, serum cholesterol, smoking habit, relative weight and ECG abnormalities to incidence of major coronary events: final report of the pooling project. The pooling project research group. J Chronic Dis. 1978 Apr;31(4):201-306. PMID:681498

2. Puska $P$, Vartiainen E. Community-based intervention studies in high-income countries. In: Detels R, Beaglehole R, Lansang MA, Gulliford M, editors. Oxford textbook of public health. Oxford: Oxford University Press; 2009:557-66.

3. Puska P, Vartiainen E, Laatikainen T, Jousilahti P, Paavola M, editors. The North Karelia Project: from North Karelia to National Action. Helsinki: Helsinki University Printing House; 2009.

4. Sarrafzadegan N, Kelishadi R, Sadri G, Malekafzali H, Pourmoghaddas M, Heidari K, et al. Outcomes of a comprehensive healthy lifestyle program on cardiometabolic risk factors in a developing country: the Isfahan Healthy Heart Program. Arch Iran Med. 2013 Jan;16(1):4-11. PMID:23273227

5. Protocol and Guidelines: Countrywide Integrated Noncommunicable Diseases Intervention (CINDI) Programme. Copenhagen: WHO Regional Office for Europe; 1996 (EUR/ ICP/CIND 94 02/PB04 (http://whqlibdoc.who.int/hq/1994/ EUR_ICP_CIND_94.02_PB04.pdf, accessed 26 June 2014).

6. WHO. Global Action Plan for the Prevention and Control of Noncommunicable diseases 2013-2020. Geneva: World Health Organization; 2013.

7. Global strategy for the prevention and control of noncommunicable diseases. Report by the Director-General (http:// apps.who.int/gb/archive/pdf_files/WHA53/ea14.pdf?ua=10, accessed 26 June 2014)
8. Tunstall-Pedoe H. Cardiovascular Risk and Risk Scores: ASSIGN, Framingham, QRISK and others: how to choose. Heart. 2011 Mar;97(6):442-4. PMID:21339319

9. Kottke TE, Puska P, Salonen JT, Tuomilehto J, Nissinen A. Projected effects of high-risk versus population-based prevention strategies in coronary heart disease. Am J Epidemiol. 1985 May;121(5):697-704. PMID:4014161

10. Political Declaration of the High-level Meeting of the General Assembly on the Prevention and Control of Non-communicable Diseases 2011 (http://www.who.int/nmh/events/ un_ncd_summit2011/political_declaration_en.pdf, accessed 26 June 2014)

11. Moran A, Tsong K, Forouzanfar M, Roth G, Mensah G, Ezzati M, et al. Variations in Ischaemic Heart Disease Burden and Risk Factors 2010 Study. Global Heart. 2014;9:91-9 (http://download.journals.elsevierhealth.com/pdfs/journals/2211-8160/ PIIS221181601300210X.pdf, accessed 26 June 2014).

12. Laatikainen T, Critchley J, Vartiainen E, Salomaa V, Ketonen M, Capewell S. Explaining the decline in coronary heart disease mortality in Finland between 1982 and 1997. Am J Epidemiol. 2005 Oct 15;162(8):764-73. PMID:16150890

13. Kontis V, Mathers CD, Rehm J, Stevens GA, Shield KD, Bonita R, et al. Contribution of six risk factors to achieving the $25 \times 25$ noncommunicable disease mortality reduction target: a modelling study. Lancet. 2014 May 2; 10.1016/s0140-6736(14)60616-4 [Epub ahead of print]. PMID:24797573 